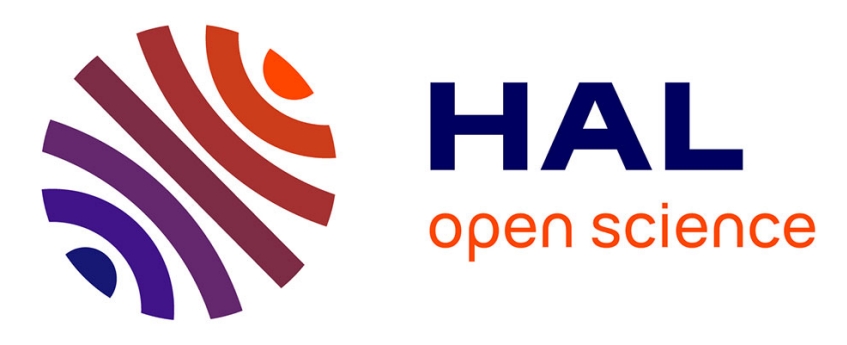

\title{
Combined Feedback Linearization and MPC for Wind Turbine Power Tracking
}

Nicolo Gionfra, Houria Siguerdidjane, Guillaume Sandou, Damien Faille, Philippe Loevenbruck

\section{- To cite this version:}

Nicolo Gionfra, Houria Siguerdidjane, Guillaume Sandou, Damien Faille, Philippe Loevenbruck. Combined Feedback Linearization and MPC for Wind Turbine Power Tracking. IEEE Multi-Conference on Systems and Control (MSC 2016), Sep 2016, Buenos Aires, Argentina. 10.1109/CCA.2016.7587821 . hal-01381416

\section{HAL Id: hal-01381416 \\ https://hal-centralesupelec.archives-ouvertes.fr/hal-01381416}

Submitted on 14 Oct 2016

HAL is a multi-disciplinary open access archive for the deposit and dissemination of scientific research documents, whether they are published or not. The documents may come from teaching and research institutions in France or abroad, or from public or private research centers.
L'archive ouverte pluridisciplinaire HAL, est destinée au dépôt et à la diffusion de documents scientifiques de niveau recherche, publiés ou non, émanant des établissements d'enseignement et de recherche français ou étrangers, des laboratoires publics ou privés. 


\title{
Combined Feedback Linearization and MPC for Wind Turbine Power Tracking *
}

\author{
Nicolò Gionfra * Houria Siguerdidjane* Guillaume Sandou* \\ Damien Faille ${ }^{* *}$ Philippe Loevenbruck ${ }^{* * *}$
* Laboratoire des Signaux et Systémes (L2S, CentraleSupélec), 3 rue Joliot Curie, 91192 Gif-sur-Yvette, France, (e-mail: \{Nicolo.Gionfra; Houria.Siguerdidjane; Guillaume.Sandou\}@centralesupelec.fr).
** EDF REDD, Department STEP, 6 quai Watier, 78401 Chatou, France, (e-mail: damien.faille@edf.fr).
*** EDF REDD, Department EFESE, 1 avenue du Général de Gaulle, 92141 Clamart, France, (e-mail: philippe.loevenbruck@edf.fr).

\begin{abstract}
The problem of controlling a variable-speed-variable-pitch wind turbine in non conventional operating points is addressed. We aim to provide a control architecture for a general active power tracking problem for the turbine's entire operating envelope. The presented control enables to cope with system non linearities while handling state and input constraints, and avoiding singular points. Simulations are carried out based on the CART turbine parameters. Comparatives results show that the proposed controller outperforms the classic PI regulator.
\end{abstract}

Keywords: Feedback linearization, predictive control, wind turbines, power tracking.

\section{INTRODUCTION}

The strong penetration of wind energy production in the distribution and transmission electric grid has changed the role that wind farms have in taking into account the grid constraints as well as in ensuring the balance between production and demand. As a result, an adapted grid code for wind power generation establishes a set of grid connection technical requirements that wind farms have to meet. These are typically related to the voltage and reactive power control, frequency control, and fault ride-through capabilities (see De Alegría et al. (2007), ENTSO-E (2013)). If in the past years, wind turbines were not expected to actively participate to the grid operations, they are nowadays required to be able to work in non conventional operating modes. On the one hand, this implies certain restrictions on the maximum power delivered to the grid, on the other hand, it opens a range of new possible services to which wind farms can participate, bringing an economical gain at the collectivity scale. This is evidenced by Delille et al. (2013) which considers the economic advantage of power curtailment as a possible alternative solution to the grid reinforcement for the integration of renewable energies. It follows a growing interest in employing new technologies, such as energy storage, and new methods of control in order to allow wind turbines to function out of the classic maximum power point tracking (MPPT) mode of operation when needed. From a technical point of view the aforementioned requirements can be expressed as constraints on active and reactive power that wind turbines have to respect while

\footnotetext{
* This study has been carried out in the RISEGrid Institute (www.supelec.fr/342p38091/risegrid-en.html), joint program between CentraleSupélec and EDF ('Electricité de France') on smarter electric grids.
}

maximizing the energy production. Furthermore, it has to be mentioned that forcing a wind turbine not to extract the maximum aerodynamic power does not directly imply a loss in the power production of the whole wind farm. In other words, the optimal power reference for a wind turbine does not necessarily coincide with the MPPT power reference if coupling effects between turbines are considered. More details can be found in Marden et al. (2013) where, in a cooperative control framework, different power set points have to be provided to each wind turbine when wake interaction is considered. Since a great deal of applications, especially concerning frequency response, active power constraints, and power optimization, can be in practice treated as a general problem of active power control, we decided to focus the work of this paper on the control of aerodynamic power extraction of variable-speed-variable-pitch turbines, i.e. the problem of tracking a desired power reference signal. This choice is validated by the possibility to control active and reactive power independently, basically thanks to power electronic converters (see Pöller (2003), Arifujjaman et al. (2009)).

As it is well known, two regions corresponding to different operating modes are distinguished in classic control of wind turbines (see Ackermann (2005)): the first one, at low wind speed, consists in the MPPT algorithm, while the second one, at high wind speed, is concerned with stabilizing the power at its nominal value. To do so, according to the current value of wind speed, references for the turbine's rotor angular speed and for the pitch angle are obtained via the static aerodynamic relation between the mentioned variables and the aerodynamic power. When the desired aerodynamic power is lower than its optimal value, different set points for the rotor angular speed and the pitch angle must be provided. Even though 
different strategies have been proposed in the literature for the choice of the latter (e.g. Yingcheng and Nengling (2011), Žertek et al. (2012)), in the most cases the control architecture is based on standard linear controllers such as PID (e.g. Loukarakis et al. (2009), Ramtharan et al. (2007), Tarnowski et al. (2009)) and gain scheduling approaches (e.g. Wang and Seiler (2014), Khezami et al. (2010), Camblong et al. (2012)). Peñarrocha et al. (2013) present a nonlinear control to let transient power increase with respect to maximum aerodynamic power to sustain the grid in the case of low wind speed and fixed pitch angle. Concerning nonlinear control techniques applied to the turbine control for MPPT mode of operation or power limiting at high wind speed, plenty of strategies have been proposed. One can cite Thomsen (2006), Boukhezzar and Siguerdidjane (2011), Boukhezzar et al. (2007). Nonetheless their extension to the more general active power control framework is not a trivial task. Moreover, to the extent of our knowledge, these approaches are conceived for well-defined modus operandi, again either MPPT or power limiting at high wind speed. Few works have treated the problem of nonlinear control for the entire operating envelope. Burkart et al. (2011) presents an approach based on switched linear systems and feedback linearization (FL) which enables the turbine to be controlled in all the regions of interest. Nevertheless, when operating at low wind speed the pitch angle is kept constant to its optimal value. This basically limits its employment for different power references than the optimal one. In this paper we present a nonlinear control for active power tracking which is not confined to work in a specific region, i.e. no assumptions were made concerning the wind speed. The employed control approach is based on a combination of FL and model predictive control (MPC) and, to the best of our knowledge, it was never applied for the sake of wind turbine control. While MPC allows dealing with state and inputs constraints explicitly, FL enables solving an optimal control problem with nonlinear constraints and whose underlying dynamic system is made linear by the FL itself. In addition, under some approximations, the optimal problem can be made convex, or even quadratic (see Nevistić and Morari (1995)). Eventually, we present a contribution concerning the treat of singular points in the FL framework. A singular point is a state of the system in which the relative degree is not well-defined. If FL technique is employed for, say, a tracking problem, and the system presents singular points, then the classic formulation of FL (Isidori (1995)) may not be applied. While in the literature approximate FL techniques, based on the work of Hauser et al. (1992), exist to overcome this problem, we propose a novel approach based on avoiding the singular points rather than performing an approximate FL.

The paper is organized as follows. In Section 2 the wind turbine dynamic model is provided. The main control problem and its objectives are stated in Section 3. In Section 4 we present the proposed control architecture for active power tracking. Simulations and comparisons with classic linear controllers are carried out in Section 5. The paper ends with conclusions and future perspectives in Section 6.

\section{WIND TURBINE MODELING}

The wind turbine model describes the conversion from wind power to electric power. The wind kinetic energy captured by the turbine is turned into mechanical energy of the turbine's rotor, turning at an angular speed $\omega_{r}$ and subject to a torque $T_{r}$. In terms of extracted power, it can be described by the nonlinear function

$$
P_{r}=\omega_{r} T_{r}=\frac{1}{2} \rho \pi R^{2} v^{3} C_{p}(\lambda, \vartheta)
$$

where $\rho$ is the air density, $R$ is the radius of the rotor's blades, $\vartheta$ is the pitch angle, $v$ is the equivalent wind speed representing the wind field impact on the turbine, obtained by filtering the time series of wind data as described by Petru and Thiringer (2002), $\lambda$ is the tip speed ratio given by $\lambda=\frac{\omega_{r} R}{v} . C_{p}$, nonlinear function of the tip speed ratio and pitch angle, is the power efficiency coefficient. This is typically provided in turbine's specifications as a look-up table. As far as the turbine's parameters are concerned, in this work we make use of the CART (Controls Advanced Research Turbine) power efficiency coefficient, shown in Fig. A.1. Nonetheless, the following analytic approximation (see Heier (1998)) is employed for the synthesis of the controller.

$$
\begin{gathered}
C_{p}(\lambda, \vartheta)=0.5\left(\frac{116}{\lambda_{i}}-0.4 \vartheta-5\right) e^{\left(\frac{-21}{\lambda_{i}}\right)}+0.0068 \lambda \\
\frac{1}{\lambda_{i}}=\frac{1}{\lambda+0.08 \vartheta}-\frac{0.035}{\vartheta^{3}+1}
\end{gathered}
$$

A drive train turns the slow rotor speed into high speed on the generator side, $\omega_{g}$. As in Burkart et al. (2011), Boukhezzar and Siguerdidjane (2011), and Thomsen (2006), we use a two-mass model represented in Fig. 1, where $J_{r}$ is the rotor inertia, $K_{s}$ is the spring constant, $D_{s}$ is the damping coefficient, $n_{g}$ the gear ratio and $J_{g}$ the generator inertia. If we neglect the generator loss, then the electric power delivered to the grid is $P_{e}=T_{g} \omega_{g}$, where $T_{g}$ is the torque applied to the generator. The implicit dynamic model is then obtained by applying the Newton's law. It follows the same system of differential equations as in Thomsen (2006), and Burkart et al. (2011)

$$
\left[\begin{array}{c}
\dot{\omega}_{r} \\
\dot{\omega}_{g} \\
\dot{\delta} \\
\dot{\vartheta} \\
\dot{T}_{g}
\end{array}\right]=\left[\begin{array}{c}
\frac{1}{J_{r}} \frac{P_{r}\left(\omega_{r}, \vartheta, v\right)}{\omega_{r}}-\frac{D_{s}}{J_{r}} \omega_{r}+\frac{D_{s}}{J_{r} n_{g}} \omega_{g}-\frac{K_{s}}{J_{r}} \delta \\
\frac{D_{s}}{J_{g} n_{g}} \omega_{r}-\frac{D_{s}}{J_{g} n_{g}^{2}} \omega_{g}+\frac{K_{s}}{J_{g} n_{g}} \delta-\frac{1}{J_{g}} T_{g} \\
\omega_{r}-\frac{1}{n_{g}} \omega_{g} \\
-\frac{1}{\tau_{\vartheta}} \vartheta+\frac{1}{\tau_{\vartheta}} \vartheta_{r} \\
-\frac{1}{\tau_{T}} T_{g}+\frac{1}{\tau_{T}} T_{g, r}
\end{array}\right]
$$

where the state $\delta$ was introduced to describe the twist of the flexible drive train. Moreover the last two equations in (3) depict the dynamic of the system's actuators: respectively the pitch angle and the generator torque actuator. Their dynamics are supposed to behave as first order model. The controlled input of the system is $u \triangleq\left[\begin{array}{ll}\vartheta_{r} & T_{g, r}\end{array}\right]^{\top}$ and its state vector is $x \triangleq$ $\left[\begin{array}{lllll}\omega_{r} & \omega_{g} & \delta & \vartheta & T_{g}\end{array}\right]^{\top}$. It is easy to see that the system is affine in the control, i.e. of the form 


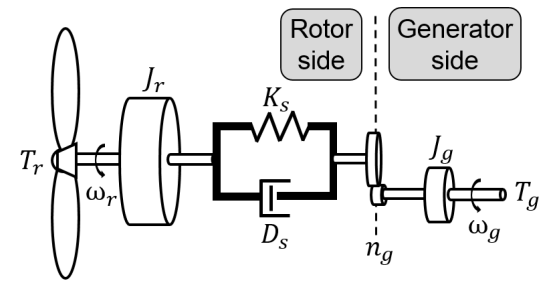

Fig. 1. Two-mass model of the turbine mechanics.

$$
\left\{\begin{array}{l}
\dot{x}=f(x, v)+g(x) u \\
P_{e}=h(x)
\end{array}\right.
$$

where $f(x, v), g(x)$ can be identified from equation (3) and $h(x) \triangleq T_{g} \omega_{g}$. Note that $v$ acts as a disturbance and it makes the system time-varying for $v$ is a function of time. The CART turbine parameters and its physical constraints are provided in table A.1.

\section{PROBLEM STATEMENT}

\subsection{Control Objectives}

In standard conditions a wind turbine is controlled to extract the maximum power from the wind when operating below the rated power and to limit it when the wind power exceeds the turbine's nominal one, $P_{e, n}$. Note that the former is given by

$$
P_{M P P T}=\max _{\left(\omega_{r}, \vartheta\right)} P_{r}\left(\omega_{r}, \vartheta, v\right)
$$

There exist though some scenarios where it would be either preferable or even compulsory to track other power references. Disregarding the reasons why the electric power reference would deviate from the classic one, we can define the main control objective as that of tracking a given electric power set point signal $P_{e}^{*}(t)$. Nonetheless, for the sake of simplicity we will consider those scenarios which involve a degradation of electric power with respect to its optimal or rated value. The reason is that even if transient set points above the maximum power would not prevent the employment of the proposed controller, they would affect the algorithm used to calculate the references for $\omega_{r}$ and $\vartheta$ corresponding to the desired $P_{e}^{*}(t)$. Being the development of such a strategy beyond the scope of this paper, we will only consider values of $P_{e}^{*}(t)$ such that

$$
\forall t \geq 0: 0 \leq P_{e}^{*}(t) \leq \min \left(P_{M P P T}, P_{e, n}\right)
$$

However we can give some realistic examples that fall within this case:

- Power optimization in a wind farm when wake effect is considered.

- Downward active power reserve.

- Constraints on maximum deliverable power, imposed by the grid operator.

Since according to (1), for a given $P_{e}^{*}<P_{M P P T}$, the choice of $\left(\omega_{r}^{*}, \vartheta^{*}\right)$ that yields $P_{e}^{*}$ is not unique, there exist different strategies to deload a wind turbine. They are typically based either on pitch control or on speed control (see Yingcheng and Nengling (2011)). The former consists in keeping $\omega_{r}$ at its optimal value $\omega_{r, o p t}$, argument of equation (4), and modifying the pitch angle. The latter involves operating the turbine at increased rotor's speed. This second approach seems to be preferable. Indeed, if the wind turbine have to be deloaded, part of the mechanical power $P_{r}$ can be used to increase the rotor speed. As a result, part of the undelivered energy to the grid can be stocked in the rotor kinetic energy

$$
\Delta W_{k}=\frac{1}{2} J_{r}\left(\omega_{r, \text { increased }}^{2}-\omega_{r, \text { opt }}^{2}\right)
$$

If then, the electric power needs to be increased again, the rotor has to slow down and its kinetic energy can be released to the grid. In this paper we make use of the strategy proposed by Žertek et al. (2012) which allows the turbine to work at an optimal operating point with respect to the amount of kinetic energy of the rotating masses. When deloading needs to be performed the set points of $\left(\omega_{r}, \vartheta\right)$ are calculated using

$$
\max \omega_{r}
$$

subject to

$$
\begin{aligned}
P_{e}^{*} & =P_{r}\left(\omega_{r}, \vartheta, v\right) \\
\omega_{r, \min } & \leq \omega_{r} \leq \omega_{r, n} \\
\vartheta_{\text {min }} & \leq \vartheta \leq \vartheta_{\max }
\end{aligned}
$$

\subsection{Problem Formulation}

Consider the system described by equation (3). Given an effective wind speed signal $v(t)$ and a time-varying reference trajectory of active power $P_{e}^{*}(t)$ verifying (5) and such that it is an admissible steady state target for system (3), i.e. $\forall t \geq 0$ it always exists an admissible solution $\left(x_{s}(t), u_{s}(t)\right)$ to the following set of nonlinear equations.

$$
\left\{\begin{array}{l}
0=f\left(x_{s}(t), v(t)\right)+g\left(x_{s}(t)\right) u_{s}(t) \\
P_{e}^{*}(t)=h\left(x_{s}(t)\right)
\end{array}\right.
$$

We can define the control problem as that of finding the input vector $u(t)$ that minimizes the distance between the system variables $(x(t), u(t))$ and the pair $\left(x_{s}(t), u_{s}(t)\right)$, $\forall t \geq 0$. Note that $(7)$ has to be solved together with the solution of (6), thus yielding a unique solution. In addition, in this paper, we assume to have access to the value of the equivalent wind speed $v$ blowing towards the turbine's axial direction, which is needed either when solving (7) and, as it is explained in Section 4, for the control design.

\section{CONTROL DESIGN}

As it has been said, the proposed controller is based on the composition of two techniques which basically divide the control design in two phases: the FL and the MPC stage.

\subsection{Feedback linearization stage}

Reproducing the work of Thomsen (2006), we employ FL to target directly the system non linearity, i.e. the first row in system (3). Indeed, since the dynamic associated to $\left[\begin{array}{lll}x_{2} & x_{3} & x_{5}\end{array}\right]^{\top}$ is already linear, this results in linearizing the relation between the input $\vartheta_{r}$ and the rotor speed $\omega_{r}$. So, if we take $x_{1}$ as system's output, it is easy to see that we need to derive it two times with respect to time in order to make the input $\vartheta_{r}$ appear. The system's relative degree associated with the output $x_{1}$ is then $r=2$. We remind that $L_{f} h(x) \triangleq \frac{\partial h}{\partial x} f(x)$. Thus, by taking the coordinate transformation

$$
\xi=T_{\xi}(x)=\left[\begin{array}{lllll}
x_{1} & L_{f} x_{1} & x_{2} & x_{3} & x_{5}
\end{array}\right]^{\top}
$$


we get the system written in the hybrid coordinates

$$
\dot{\xi}=A_{\xi} \xi+B_{\xi}\left(f_{\xi}(x)+g_{\xi}(x) u\right)
$$

where the pair $\left(A_{\xi}, B_{\xi}\right)$ is controllable. Note that system (9) is not in the normal form, yet FL can still be applied (see Khalil (2002)). The main advantage of transformation (8) is that the variables in the new coordinates preserve a physical meaning and this is helpful when designing a controller in cascade with FL such that the closed loop system has desired performance. At this point we make the particular choice to use FL to only eliminate the non linearities of the system, which are concentrated in the second row of system (9). In particular we can write the latter as

$$
\begin{aligned}
\dot{\xi}_{2} & =L_{f}^{2} x_{1}+L_{g} L_{f} x_{1} u \\
& =\alpha(\xi, \vartheta, v, \dot{v})+a_{2} \xi+\beta(\xi, \vartheta, v) \vartheta_{r}
\end{aligned}
$$

where $a_{2}$ is a row vector such that $a_{2} \xi$ collects all the linearities in $L_{f}^{2} x_{1}, \alpha(\xi, \vartheta, v, \dot{v})+a_{2} \xi=L_{f}^{2} x_{1}$ and $\beta(\xi, \vartheta, v) \vartheta_{r}=L_{g} L_{f} x_{1} u$. Thus the feedback linearizing input can be chosen as

$$
\vartheta_{r, F L} \triangleq \vartheta_{r}=\frac{1}{\beta(\xi, \vartheta, v)}\left(-\alpha(\xi, \vartheta, v, \dot{v})+v_{\vartheta}\right)
$$

where $v_{\vartheta}$ is left as degree of freedom as in classic FL technique. The feedback linearized system is then

$$
\begin{aligned}
& \dot{\xi}=A \xi+B\left[\begin{array}{ll}
v_{\vartheta} & T_{g, r}
\end{array}\right]^{\top} \\
& =\left[\begin{array}{ccccc}
0 & 1 & 0 & 0 & 0 \\
a_{2,1} & 0 & a_{2,3} & a_{2,4} & a_{2,5} \\
\frac{D_{s}}{n_{g} J_{g}} & 0 & -\frac{D_{s}}{n_{g}^{2} J_{g}} & \frac{K_{s}}{n_{g} J_{g}} & -\frac{1}{J_{g}} \\
1 & 0 & -\frac{1}{n_{g}} & 0 & 0 \\
0 & 0 & 0 & 0 & -\frac{1}{\tau_{T}}
\end{array}\right] \xi+\left[\begin{array}{cc}
0 & 0 \\
1 & 0 \\
0 & 0 \\
0 & 0 \\
0 & \frac{1}{\tau_{T}}
\end{array}\right]\left[\begin{array}{c}
v_{\vartheta} \\
T_{g, r}
\end{array}\right]
\end{aligned}
$$

where the pair $(A, B)$ is controllable. The choice of $(10)$ is motivated by two main reasons. The first is that we want to reduce the number of exact cancellations as they are intrinsically non robust, leaving them just to the non linear terms. Secondly, in this application, we want to avoid non interactive control. Indeed if the term $a_{2} \xi$ was canceled by FL control, then the variable $\xi_{1}$, which is the rotor's speed $\omega_{r}$ in the original coordinates, would be controlled only by $v_{\vartheta}$. However, being $\vartheta_{r}$ limited in order to limit $\vartheta$ (see table A.1), this in turns results in constraints on $v_{\vartheta}$. Thus if we let decoupling, we implicitly affect the controllability of the system. Being $a_{2}$ a nonzero row, we are able to keep a connection between the input $T_{g, r}$ and the variable $\xi_{1}$.

\subsection{Model predictive control stage}

We now have to design a controller for linear system (11). The choice of MPC is mainly motivated by its capability to explicitly handle state and input constraints. As a matter of fact system (11) is subject to the following constraints

$$
\begin{aligned}
\omega_{r, \min } & \leq \xi_{1} \leq \omega_{r, \max } \\
0 & \leq \xi_{3} \\
0 & \leq \xi_{5} \\
\vartheta_{\min } & \leq \vartheta_{r, F L} \leq \vartheta_{\max }
\end{aligned}
$$

A few words have to be spent on the last inequality of (12). Since we are not able to explicitly inverse the transformation $T_{\xi}(x)$, we cannot use an analytic relation between $\vartheta$ and the new coordinates $\xi$. This is the reason why we choose to limit $\vartheta_{r}$ in order to respect the constraints on $\vartheta$. Even though this solution is conservative, if the constraints on $\vartheta_{r}$ are satisfied, so they will be on $\vartheta$ through the fourth equation of (3).

In this framework we make use of MPC to treat also another problem, namely the one of avoiding the singular points. Before getting insight into the proposed solution, it is useful to provide the following proposition.

Proposition 1. Consider a SISO system of the form

$$
\left\{\begin{array}{l}
\dot{x}=f(x)+g(x) u, \quad x(0)=x_{0} \\
y=h(x)
\end{array}\right.
$$

where $x \in \Omega \subseteq \mathbb{R}^{n}$. Then $L_{g} L_{f}^{r-1} h(x(t)) \neq 0 \quad \forall t \geq 0$, i.e. the trajectory $x(\cdot)$ does not pass through singularities if and only if the following conditions are verified.

c.1.1 The system's relative degree in $x_{0}$ is well-defined and equal to $r \leq n$.

c.1.2 $\operatorname{sign}\left(L_{g} L_{f}^{r-1} h(x(t))\right)=\operatorname{sign}\left(L_{g} L_{f}^{r-1} h\left(x_{0}\right)\right) \forall t \geq 0$.

Proof.(Necessity) If for some $t^{*}>0, \operatorname{sign}\left(L_{g} L_{f}^{r-1} h\left(x\left(t^{*}\right)\right)\right)$ $\neq \operatorname{sign}\left(L_{g} L_{f}^{r-1} h\left(x_{0}\right)\right)$, then $\exists t^{* *}: 0<t^{* *} \leq t^{*}$ such that $L_{g} L_{f}^{r-1} h\left(x\left(t^{* *}\right)\right)=0$, i.e. $x\left(t^{* *}\right)$ is a singular point. (Sufficiency) If for some $\left.t^{*}>0, L_{g} L_{f}^{r-1} h\left(x\left(t^{*}\right)\right)\right)=0$ then, by condition c.1.2, $L_{g} L_{f}^{r-1} h\left(x_{0}\right)=0$ and this contradicts condition c.1.1.

Note that if the system trajectory is the solution of the closed loop system where $u$ is chosen as the FL control $u=$ $\left(L_{g} L_{f}^{r-1} h(x)\right)^{-1}\left(-L_{f}^{r} h(x)+v\right)$, then, if the conditions of proposition 1 are verified, $u$ is bounded. Proposition 1 gives simple conditions under which a given trajectory of the state does not pass through points where the accessibility of the system is lost. A natural question is under which conditions such a trajectory exists. In particular we are interested in finding a trajectory connecting two given points while satisfying the conditions of proposition 1 .

Fact 2. Consider system (13), where $x_{0}$ respects condition c.1.1. Consider a given output $y_{e}$ and a corresponding equilibrium pair $\left(x_{e}, u_{e}\right)$, i.e. such that $0=f\left(x_{e}\right)+g\left(x_{e}\right) u_{e}$, $h\left(x_{e}\right)=y_{e}$. If the following conditions are verified

c.2.1 The system's relative degree in $x_{e}$ is well-defined.

c.2.2 $\operatorname{sign}\left(L_{g} L_{f}^{r-1} h\left(x_{e}\right)\right)=\operatorname{sign}\left(L_{g} L_{f}^{r-1} h\left(x_{0}\right)\right)$.

c.2.3 By defining the set $\Lambda \triangleq\left\{x \in \Omega: \operatorname{sign}\left(L_{g} L_{f}^{r-1} h(x)\right)\right.$ $\left.=\operatorname{sign}\left(L_{g} L_{f}^{r-1} h\left(x_{0}\right)\right)\right\}, x_{0}, x_{e}$ belong to a connected subset of $\Lambda: \Lambda_{c}$.

Then, by definition of connected set, it always exists a curve $\gamma$ such that $x_{0}, x_{e} \in \gamma$ and $\gamma \in \Lambda_{c}$, which implies $L_{g} L_{f}^{r-1} h(x) \neq 0 \quad \forall x \in \gamma$.

Fact 2 states that, under particular conditions, among all the possible curves connecting two given points, there always exists one which does not pass through singular points. Note that the conditions of fact 2 are necessary for the existence of a trajectory connecting two given points while avoiding singular points, i.e. a curve satisfying the conditions of fact 2 and system's equations (13). 


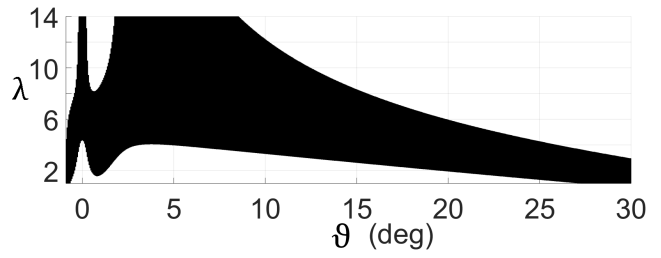

Fig. 2. $\Lambda_{t}$ : set of $(\lambda, \vartheta)$ such that $\beta(\lambda, \vartheta)<0$.

The relative degree of the turbine (3) with respect to the output $x_{1}$ is not global. Namely, there exist points where $\beta(\xi, \vartheta, v)=0$. Nonetheless, by simulation, $\beta$ appears to be negative-valued in the point of functioning of interest. In addition, numerical analysis shows that the domain in which $\beta$ has negative value is connected, provided that $\vartheta>$ -1 . Indeed $\vartheta=-1$ is a singularity for $(2)$, so we limit $\vartheta$ to the range $\left(-1, \vartheta_{\max }\right]$. Moreover $\beta(\xi, \vartheta, v)=\beta\left(\xi_{1}, \vartheta, v\right)$, i.e. it only depends on the variables $\xi_{1}, \vartheta$, and $v$ and it can further reduced to $\beta(\lambda, \vartheta)$. This allow us to show that the couples $(\lambda, \vartheta)$ such that $\beta<0$, in the domain of interest, form a connected set. We name the latter $\Lambda_{t}$, (see Fig. 2). By employing MPC, then, we have the tools to fall within the conditions of proposition 1 and fact 2 and use (10) to feedback linearize the system while avoiding singular points.

We consider a discrete MPC for the discretization of linear system (11). In the classic MPC formulation, at each time step a new steady state target is computed and it is kept constant during the whole prediction horizon. The control input is then calculated for the entire horizon based on the current state of the system and the steady state target. Only its first value is applied to the system. The process reiterates at the next step based on the new current state of the system and the new target. Provided that the system initial state satisfies $c .1 .1$ and belongs to $\Lambda_{t}$, we only need to verify that the target state belongs to $\Lambda_{t}$ too at each time step and to find a control that imposes $c$.1.2. In order to calculate the steady state target, note that $\xi$ has a clear physical meaning thanks to the choice of (8), and it is easy to find a steady state solution $\left(\xi_{s}, v_{\vartheta, s}, T_{g, r, s}\right)$ for a given $P_{e}^{*}$ and $\omega_{r}^{*}$, (argument of $(6)$ ). Indeed, instead of solving the system of nonlinear equations (7), one can use

$$
\left\{\begin{array}{l}
A \xi_{s}+B\left[v_{\vartheta, s} \quad T_{g, r, s}\right]^{\top}=0 \\
\xi_{3, s} \xi_{5, s}=P_{e}^{*} \\
\xi_{1, s}=\omega_{r}^{*}
\end{array}\right.
$$

As far as the control is concerned, we impose the following additional constraint to (12) so that c.1.2 is satisfied for the whole prediction horizon.

$$
\beta(\xi, \vartheta, v)+\varepsilon<0, \quad \varepsilon \geq 0
$$

If (15) is satisfied then the new state is guaranteed to lie in $\Lambda_{t}$. Note that we added a margin $\varepsilon$ to $(15)$, because model-plant mismatch and disturbances may make the actual new state to lie outside $\Lambda_{t}$ and this would imply the passage through a singular point.

We can now provide the MPC problem formulation which leads to the implicit control law $\kappa(\cdot): \mathbb{R}^{8} \rightarrow \mathbb{R}^{2}$ such that $u_{M P C} \triangleq\left[\begin{array}{ll}v_{\vartheta} & T_{g, r}\end{array}\right]^{\top}=\kappa(\xi, \vartheta, v, \dot{v})$. The optimization problem that has to be solved at each time step $j$ is defined as follows.

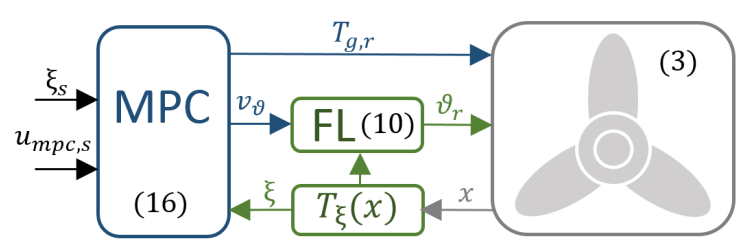

Fig. 3. FL + MPC scheme.

$$
\begin{aligned}
\min _{\left\{s, u_{M P C}\right\}} \sum_{k=1}^{N-1}\left(\xi_{s}-\xi(k)\right)^{\top} Q_{\xi}\left(\xi_{s}-\xi(k)\right)+z(k)^{\top} Q_{z} z(k) \\
+\left(u_{M P C, s}-u_{M P C}(k)\right)^{\top} R\left(u_{M P C, s}-u_{M P C}(k)\right)+r_{s} s(k)^{2} \\
+\Delta u_{M P C}(k)^{\top} R_{\Delta} \Delta u_{M P C}(k)+\left(\xi_{s}-\xi(N)\right)^{\top} P\left(\xi_{s}-\xi(N)\right) \\
\text { subject to } \\
\bullet \text { discretization of }(11) \\
\bullet(12) \\
\bullet(15) \\
\bullet \xi_{3} \xi_{5} \leq 1.05 \cdot P_{e, n}+s, \quad s \geq 0
\end{aligned}
$$

where we named $u_{M P C, s} \triangleq\left[\begin{array}{ll}v_{\vartheta, s} & T_{g, r, s}\end{array}\right]^{\top},\left(\xi_{s}, u_{M P C, s}\right)$ is the solution of (14), $\Delta u_{M P C}(k) \triangleq u_{M P C}(k)-u_{M P C}(k-1)$, $N$ is the prediction horizon, $Q_{\xi} \geq 0, Q_{z}, R, R_{\Delta}>0$ are the weight matrices, and $P$ is the positive definite matrix solution of the Riccati equation that solves the infinite-horizon LQR problem for the discretization of system (11). In addition we added a constraint (the last in (16)) to limit the maximum power to $1.05 \cdot P_{e, n}$. In order to turn the latter in a soft constraint and to avoid problem infeasibility, we also added a slack variable $s$ to the optimization problem and the corresponding weight $r_{s}$. Furthermore, we enriched the MPC problem with an integral action, represented by the variable $z$, on the errors on $\xi_{3}$ and $\xi_{5}$. This helps to keep the error on $P_{e}$ bounded in presence of disturbances and model-plant mismatch. For instance, a source of error is given by the mismatch between (2) and the actual $C_{p}$ of Fig. A.1. Note that because of non-convex state dependent constraints in (16) the optimization problem is inherently non-convex. However we can perform some approximations that render the optimization problem convex (see Nevistić and Morari (1995)). In this paper we apply a linear approximation of the constraints, updated at each time step $j$ in the current state, thus yielding the constraints of the form

$$
F_{j} \xi+G_{j} u_{M P C} \leq b_{j}
$$

where the matrices $F_{j}, G_{j}$, and $b_{j}$ represent the linear approximation at the current time step $j$, i.e. valued in the current state. Equation (17) describes a time-varying polytope, which is kept constant during the prediction horizon, and it makes the optimization problem quadratic.

\subsection{FL and MPC composition}

The overall controller is composed as follows (see Fig. 3).

- A first FL stage is employed to linearize the system via equation (10).

- Constraints on MPC either assure the physical constraints of the turbine to be respected and prevent the system trajectories to pass through singular points.

- Boundedness of the error in the state is assured by the closed-loop MPC. Proof of stability is not provided in this paper though. 




Fig. 4. Effective wind speed signal $v$.

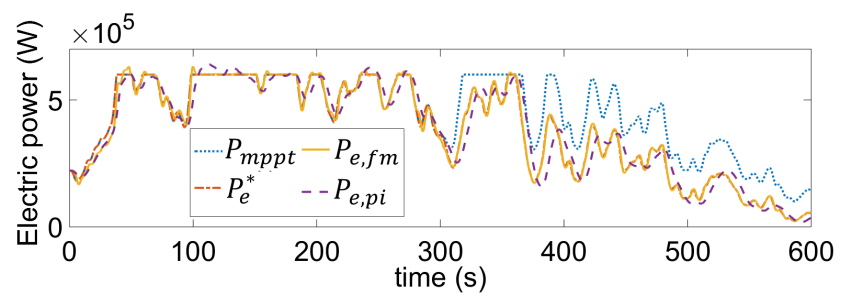

Fig. 5. Electric power signals.

\section{SIMULATION RESULTS}

We compare the proposed controller with a PI. The latter is composed of two separate feedback loops. The first one is concerned with the control of $\omega_{r}$ by acting on $T_{g, r}$, which is the output of a PI controller. The second one enables power limiting through the use of the pitch angle. Thus $\vartheta_{r}$ is output of a PI controller comparing the power reference and the actual mechanical power. This controller is activated only when the wind power is higher than the reference one. $\vartheta_{r}$ is kept constant to its optimal MPPT value otherwise.

As far as the simulation is concerned, during $600 \mathrm{~s}$ the turbine is excited by the effective wind speed in the axial direction shown in Fig. 4. For the first $300 \mathrm{~s}$ the turbine is controlled in the classic functioning of MPPT and power limiting, proving the capability of the proposed controller to work in the entire operating envelope. After $300 \mathrm{~s}$ the turbine is deloaded of a certain time-varying factor with respect to the maximum extractible power, as shown in Fig. 5, where the reference power signal $P_{e}^{*}$ (red dashed-dotted line) drifts from the maximum power $P_{m p p t}$ (blue dotted line). The $\omega_{r}$ reference corresponding to $P_{e}^{*}$ is calculated via (6). As shown in Fig. 6, it reaches the nominal value $\omega_{r, n}$ (red dashed-dotted line), drifting from $\omega_{r, m p p t}$ (blue dotted line) which corresponds to the $P_{m p p t}$ signal. FL-MPC $(f m)$ and PI $(p i)$ responses are compared throughout the whole simulation time (see Fig. 5 and Fig. 6). The main results are reported in table 1 and they show that even if the delivered electrical energy is comparable, FL-MPC outperforms the PI in terms of precision, leading to smaller errors on the desired power and rotor speed. In addition Fig. 7 shows how during the entire simulation, constraint (15) is satisfied yielding negative values of $\beta$ in the system trajectories. Eventually, Fig. 8 and Fig. 9 show the pitch angle and the generator torque signal respectively.

Table 1. 600 s Simulation on FL-MPC vs PI

\begin{tabular}{llll} 
& Energy & $\overline{\left|e r \omega_{\omega_{r}}\right|}$ & $\overline{\left|e r r_{P_{e}}\right|}$ \\
\hline FL-MPC & $232.51 \mathrm{MJ}$ & $0.0434 \mathrm{rad} / \mathrm{s}$ & $8.315 \mathrm{~kJ}$ \\
PI & $231.79 \mathrm{MJ}$ & $0.1420 \mathrm{rad} / \mathrm{s}$ & $36.884 \mathrm{~kJ}$ \\
FL-MPC/PI & 1.0031 & 0.3059 & 0.2254 \\
\hline
\end{tabular}



Fig. 6. Rotor speed signals.

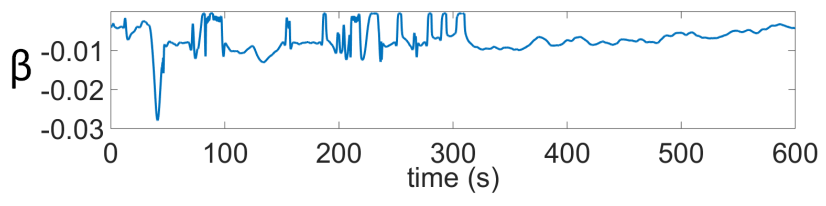

Fig. 7. Value of $\beta(\xi, \vartheta, v)$ in the system trajectories.

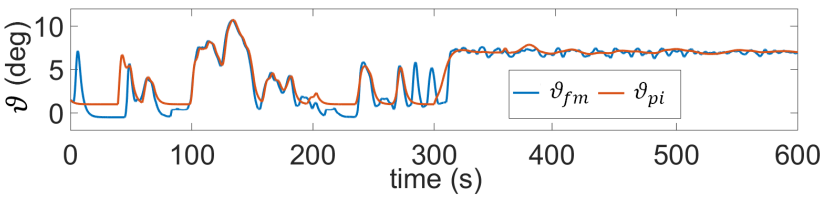

Fig. 8. $\vartheta$ signals.

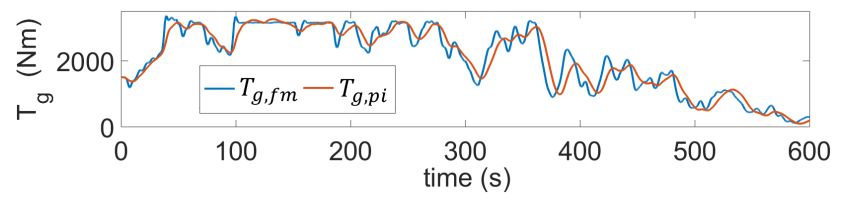

Fig. 9. $T_{g}$ signals.

\section{CONCLUSION}

A novel approach to control a wind turbine for the general problem of tracking a given power reference was presented. Composing the two well-known techniques of FL and MPC showed clear benefit when treating a nonlinear system subject to physical constraints such as the wind turbine. The proposed controller gains in precision when compared to classic linear controllers. It inherits the need for weight tuning from the MPC technique though. A proof of stability was not provided and it is object of near future work. Also, it is necessary to test robustness in the case of uncertain model parameters and unmeasured variables such as the wind speed. Indeed we make explicit use of the knowledge of the wind speed and its first time derivative. Finally we aim to employ the presented architecture for the sake of controlling a wind park.

\section{REFERENCES}

Ackermann, T. (2005). Wind power in power systems. John Wiley and Sons Ltd.

Arifujjaman, M., Iqbal, M. T., and Quaicoe, J. E. (2009). Vector control of a DFIG based wind turbine. Journal of Electrical $\&$ Electronics Engineering, volume 2, 10571066 .

Boukhezzar, B., Lupu, L., Siguerdidjane, H., and Hand, M. (2007). Multivariable control strategy for variable speed, variable pitch wind turbines. Renewable Energy, volume $32,1273-1287$. 
Boukhezzar, B., and Siguerdidjane, H. (2011). Nonlinear Control of a Variable-Speed Wind Turbine Using a TwoMass Model. Energy Conversion, IEEE Transactions on, volume 26, 149-162.

Burkart, R., Margellos, K., and Lygeros, J. (2011). Nonlinear control of wind turbines: An approach based on switched linear systems and feedback linearization. Decision and Control and European Control Conference (CDC-ECC), 2011 50th IEEE Conference on, 54855490 .

Camblong, H., Nourdine, S., Vechiu, I., and Tapia, G. (2012). Control of wind turbines for fatigue loads reduction and contribution to the grid primary frequency regulation. Energy, volume 48, 284-291.

De Alegría, I. M., Andreu, J., Martín, J. L.,Ibañez, P., Villate, J. L., and Camblong, H. (2007). Connection requirements for wind farms: A survey on technical requirements and regulation. Renewable and Sustainable Energy Reviews, volume 11, 1858-1872.

Delille, G., Malarange, G., and Gaudin, C. (2013). Analysis of the options to reduce the integration costs of renewable generation in the distribution networks. Part 2: A step towards advanced connection studies taking into account the alternatives to grid reinforcement. Electricity Distribution (CIRED 2013), 22nd International Conference and Exhibition on, 1-4.

ENTSO-E. (2013). Network Code for Requirements for Grid Connection Applicable to all Generators. European Network of Transmission System Operators for Electricity (ENTSO-E), 1-85.

Hauser, J., Sastry, S., and Kokotovic, P. (1992). Nonlinear control via approximate input-output linearization: the ball and beam example. IEEE transactions on automatic control,volume 37, 392-398.

Heier, S. (1998). Grid integration of wind energy conversion systems. Wiley.

Isidori, A. (1995). Nonlinear control systems. Springer Science \&f Business Media.

Khalil, H. K. (2002). Nonlinear systems (Third ed.) Prentice Hall.

Khezami, N., Braiek, N. B., and Guillaud, X. (2010). Wind turbine power tracking using an improved multimodel quadratic approach. $\{I S A\}$ Transactions, volume 49, $326-334$.

Loukarakis, E., Margaris, I., and Moutis, P. (2009). Frequency control support and participation methods provided by wind generation. Electrical Power Energy Conference (EPEC), 2009 IEEE, 1-6.

Marden, J. R., Ruben, S. D., and Pao, L. Y. (2013). A Model-Free Approach to Wind Farm Control Using Game Theoretic Methods. Control Systems Technology, IEEE Transactions on, volume 21, 1207-1214.

Nevistić, V., and Morari, M. (1995). Constrained Control of Feedback-Linearizable Systems. Proceedings of $3 \mathrm{rd}$ European Control Conference, volume 3, 1726-1731.

Peñarrocha, I., Dolz, D., Aparicio, N., and Sanchis, R. (2013). Synthesis of nonlinear controller for wind turbines stability when providing grid support. International Journal of Robust and Nonlinear Control, volume 24, 2261-2284.

Petru, T., and Thiringer, T. (2002). Modeling of wind turbines for power system studies. Power Systems, IEEE Transactions on, volume 17, 1132-1139.
Pöller, M. A. (2003). Doubly-fed induction machine models for stability assessment of wind farms. Power Tech Conference Proceedings, 2003 IEEE Bologna, volume 3, 6.

Ramtharan, G., Ekanayake, J. B., and Jenkins, N. (2007). Frequency support from doubly fed induction generator wind turbines. Renewable Power Generation, IET, volume $1,3-9$.

Tarnowski, G. C., Kjar, P. C., Sorensen, P. E., and Ostergaard, J. (2009). Variable speed wind turbines capability for temporary over-production. Power Energy Society General Meeting, 2009. PES '09. IEEE, 1-7.

Thomsen., S. C. (2006). Nonlinear Control of a Wind Turbine. Thesis, Informatics and Mathematical Modelling, Technical University of Denmark, DTU.

Wang, S. and Seiler, P. J. (2014) Gain Scheduled Active Power Control for Wind Turbines. 32nd ASME Wind Energy Symposium, AIAA SciTech.

Yingcheng, X., and Nengling, T. (2011). Review of contribution to frequency control through variable speed wind turbine. Renewable Energy, volume 36, 1671-1677.

Žertek, A., Verbič, G., and Pantoš, M. (2012). Optimised control approach for frequency-control contribution of variable speed wind turbines. Renewable Power Generation, IET, volume 6, 17-23.

\section{Appendix A. CART TURBINE PARAMETERS}

Table A.1. CART Turbine parameters

\begin{tabular}{lll} 
Parameter & Value & Units \\
\hline$R$ & 21.65 & $\mathrm{~m}$ \\
$n_{g}$ & 43.165 & \\
$K_{s}$ & 269.1 & $\mathrm{kN} \cdot \mathrm{m} / \mathrm{rad}$ \\
$D_{s}$ & 9500 & $\mathrm{~N} \cdot \mathrm{m} / \mathrm{rad} / \mathrm{s}$ \\
$J_{r}$ & $3.25 \cdot 10^{5}$ & $\mathrm{~kg} \cdot \mathrm{m}^{2}$ \\
$J_{g}$ & 34.4 & $\mathrm{~kg} \cdot \mathrm{m}^{2}$ \\
$\omega_{r, n}$ & 4.3982 & $\mathrm{rad} / \mathrm{s}$ \\
$\omega_{r, \max }$ & 5.5501 & $\mathrm{rad} / \mathrm{s}$ \\
$\vartheta_{\max }$ & 30 & $\mathrm{deg}$ \\
$\vartheta_{\text {min }}$ & -5 & $\mathrm{deg}$ \\
$|\dot{\vartheta}|_{\text {max }}$ & 19 & $\mathrm{deg} / \mathrm{s}$ \\
$T_{r, \max }$ & 162 & $\mathrm{kN} \cdot \mathrm{m}$ \\
$P_{e, n}$ & 600 & $\mathrm{~kW}$ \\
\hline
\end{tabular}

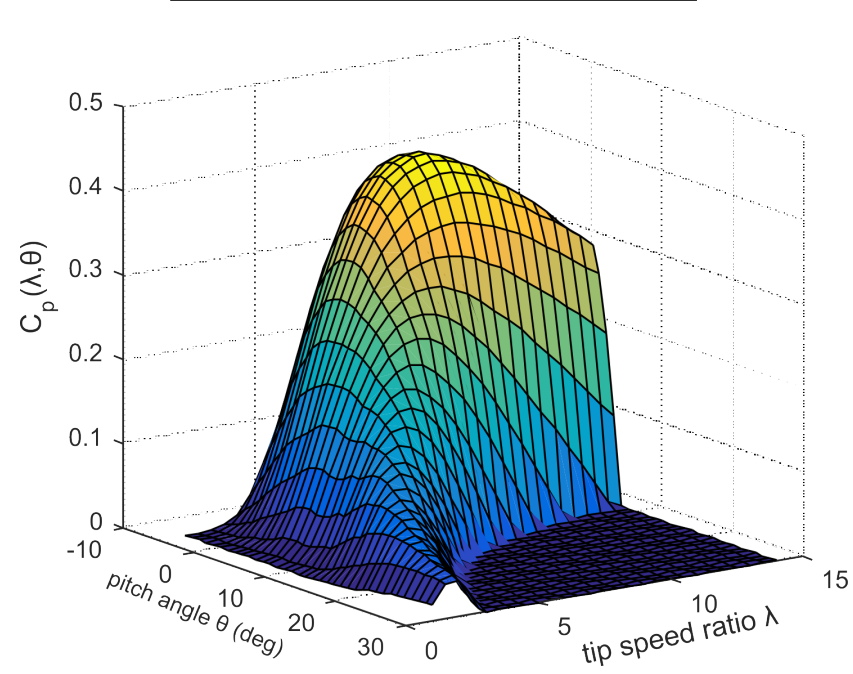

Fig. A.1. CART power efficiency coefficient $C_{p}$. 